can be associated with significant morbidity and mortality related to systemic involvement or treatment-related complications.

Objectives: This study aimed to describe the clinical features and survival of patients with inflammatory myositis in our centre.

Methods: We performed a single-centre, retrospective study on patients with inflammatory myositis (polymyositis [PM], dermatomyositis [DM], or Anti-Jo1 syndrome) seen from 2000 to 2014, noting their demographic data, clinical features and outcome until December 2016. The primary outcome assessed was all-cause mortality. Cumulative mortality rates were estimated using the KaplanMeier test; the Log Rank (Mantle-Cox) test was used to compare subgroup differences in survival.

Results: Seventy-four patients (19 PM, 28 DM, 27 Anti-Jo1 syndrome) were available for the study. Median age at diagnosis was 47 years (min 17, max 75) and median follow-up time was 93 months (min 4, max 311). Sixty-one patients $(82,4 \%)$ were female and $13(17,6 \%)$ were male. Malignancy was found in 4 patients ( 2 invasive ductal adenocancer of breast, 1 over cancer and 1 non-small cell lung cancer) and they were all female DM patients. Nineteen patients $(25,7 \%)$ died at the end of the follow-up.

The 19 patients with PM consisted of 15 female and 6 male, with a median age at diagnosis of 45 years ( $\min 22, \max 74$ ) and median follow-up time of 88 months ( $\min 5$, max 204). The 28 patients with DM consisted of 24 female and 4 male, with a median age at diagnosis of 52 years $(\min 17, \max 75)$ and median follow-up time of 80 months ( $\min 4, \max 288$ ). The 27 patients with Anti-Jo1 syndrome consisted of 22 female and 5 male, with a median age at diagnosis of 50 years ( $\min 22$, max 63 ) and median follow-up time of 117 months ( $\min 5$, max 311).

Overall survival rates of the whole group were $91 \%, 83 \%$, and $76 \%$ for 1,5 , and 10 years, respectively. The survival rates at 1,5 and 10 years from the diagnosis were respectively $88 \%, 82 \%$, \%82 for PM, \%88, $80 \%, \% 80$ for DM and $96 \%, 88 \%, 74,5 \%$ for Anti-Jo1 syndrome. But there was no significant difference between the survival rates of the diagnose groups $(p=0,734)$. Also there wasn't a significant difference between the survival rates of sex and age groups $(p=0,503)$, $(p=0,112)$. But the survival rates were significantly lower in patients with the time from diagnose less than 8 years $(p=0,000)$.
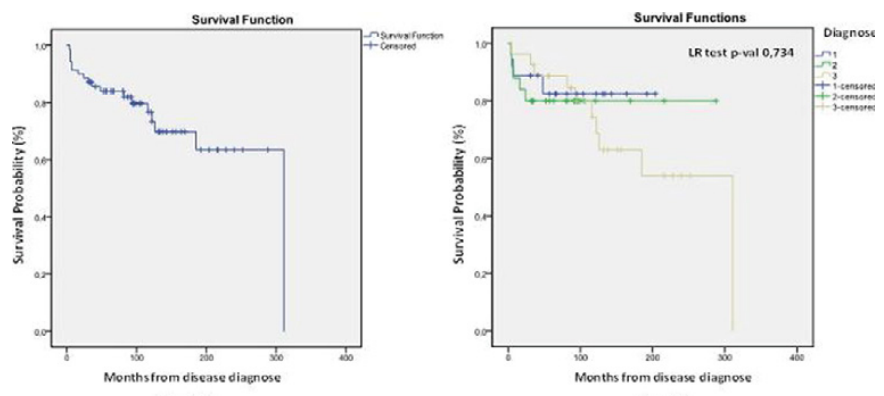

Groph A

Groph

Figure 1. Survival probability of all patients with inflamm

Conclusions: Our study involved 74 patients followed up for a median of 7,7 years and is one of the largest cohorts of patients with inflammatory myositis in Turkey. Survival was quite similar with the literature. Usually mortality has been expected in the first years after the diagnosis.

Disclosure of Interest: None declared

DOI: 10.1136/annrheumdis-2017-eular.5956

\section{SAT0339 LUNG TRANSPLANTATION IN PATIENTS WITH INTERSTITIAL LUNG DISEASE ASSOCIATED WITH ANTISYNTHETASE AND ANTI-MDA5 SYNDROMES. EXPERIENCE FROM A REFERENCE SPANISH HOSPITAL}

E. Trallero-Araguás ${ }^{1}$, C. Berastegui ${ }^{2}$, M. López-Corbeto ${ }^{1}$, I. Bello ${ }^{3}$, A. Román ${ }^{2}$, A. Selva-O'Callaghan ${ }^{4} .{ }^{1}$ Rheumatology Unit; ${ }^{2}$ Pneumology Department; ${ }^{3}$ Thoracic Surgery Department; ${ }^{4}$ Internal Medicine Department, Hospital Vall d'Hebron, Barcelona, Spain

Background: Interstitial lung disease (ILD) is the most characteristic feature and prognosis determinant of patients with antisynthetase (AS) and anti-MDA5 syndromes. Despite immunosuppressive treatment, ILD sometimes progresses to an end-stage lung disease, for which lung transplantation (LT) is the only therapeutic option. There is scarce data about post-LT outcome in this group of patients.

Objectives: To describe clinical characteristics and post-LT outcome of patients with ILD associated to AS and anti-MDA5 syndromes included in the LT program of the Vall d'Hebron Hospital of Barcelona.

Methods: We performed a review of patient records listed in the LT program. Results: From 1990 to 2016 ten patients (5 women) with ILD related to AS or anti-MDA5 syndromes were included in the LT program ( $3 \%$ of patients with ILD accepted for LT). Nine patients (2 anti-MDA5, 4 anti-Jo1, 2 anti-PL12 and 1 antiPL7) received LT while 1 patient (anti-Jo1) was still in list at the end of this study. Median age (range) of disease diagnosis was 39 years (25-55). Six patients had clinical myopathy [2 dermatomyositis (DM) and 4 polymyositis] whereas 1 patient was diagnosed with amyopathic DM. Four patients had associated pulmonary hypertension. Time between disease diagnosis and patient inclusion in LT list was higher in anti-Jo1 patients [median (range) 8.8 years (8.3-17.6)] than in the rest of the cohort [anti-MDA5 $<1$ year; antiPL12 0.7 and 3.9 years; anti-PL7 1.3 years] $(p<0.05)$. Four patients underwent bilateral LT and 5 unilateral LT. Three patients received an urgent LT (2 anti-MDA5 and 1 anti-Jo1). Six patients presented an histologic pattern of UIP and 3 of NSIP. Early complications ( $<45$ days) included: primary graft disfunction in 3 patients; phrenic paresis in 2 patients and infection in 6 patients. One patient suffered an acute rejection (AR). Six patients presented late complications ( $>45$ days): one patient developed an acute cellular rejection (ACR) and an autoantibody mediated rejection 8 and 10 months from LT respectively; 4 patients suffered chronic lung allograft dysfunction (CLAD), 2 of whom are still alive; Infection was diagnosed in 6 patients. One patient developed a squamous skin carcinoma. No flare of myopathic or lung disease was observed after LT in any case. Four patients (44\%) died: 2 of an acute respiratory failure in the immediate postoperatory period ( 1 caused by suture dehiscence and 1 by refractory ACR); 1 of an invasive aspergillosis, 17 months after LT; and 1 of CLAD after 54 months. Median follow-up (range) of the rest of the cohort was 35 months (8-70). All patients who survived more than 45 days recovered an optimal functional capacity for daily activities with no request for long-term oxygen therapy. Conclusions: LT should be considered a valid option to treat patients with end-stage or severe and rapidly progressive ILD associated to AS and anti-MDA5 syndromes. An early remission to LT referral centers for evaluation should be considered especially in non-Jo1 patients. No relapse of myositis or ILD was observed after LT. Mortality could not be attributed to the primary disease.

Disclosure of Interest: None declared

DOI: 10.1136/annrheumdis-2017-eular.6320

\section{SAT0340 SUBCLINICAL CARDIOVASCULAR DISEASE IN SCLERODERMA: A STUDY WITH CARDIOVASCULAR RISK CHARTS, CT CORONARY CALCIUM SCORE AND CAROTID ULTRASONOGRAPHY}

I. Sanz Pérez ${ }^{1}$, F. Martínez Valle $^{1}$, A. Guillén del Castillo ${ }^{1}, \mathrm{~N} . \mathrm{Pizzi}^{2}$, A. Roque Pérez $^{3}, H$. Cuéllar Calàbria ${ }^{3}, A$. Fernández Codina ${ }^{1}$, E. Callejas Moragas ${ }^{1}$ V. Fonollosa Pla ${ }^{1}$, C.P. Simeón Aznar ${ }^{1} .{ }^{1}$ Internal Medicine; ${ }^{2}$ Nuclear cardiology; ${ }^{3}$ Radiology, Hospital Universitari Vall d'Hebron, Barcelona, Spain

Background: Recently published population-based cohort studies had shown a high prevalence of cardiovascular (CV) disease in Systemic Sclerosis (SSc) patients.

Objectives: The aim of this study is to compare three different methods to measure CV risk in patients with scleroderma.

Methods: We conducted a cross-sectional study in a single center that included 43 SSc patients without CV events. We used both CV risk assessment charts SCORE for populations with low CV risk and REGICOR algorithm adjusted to Spanish subjects. Coronary Compute Tomography (CT) with coronary arterial calcium score (CACscore) was performed considering several cut-off points as predictors of CV risk. Carotid doppler ultrasound was performed to measure the Carotid Intima Media Thickness (CIMT) and for the detection of cholesterol plaques, according to Mannheim consensus criteria.

Results: Risk factors and SSc related features are described in table 1 None of the patients were catalogued as high risk according to SCORE chart (>5\%). According to REGICOR chart, 17 patients $(39,5 \%)$ were catalogued as intermediate risk and none as high risk. Twenty-two patients $(51,2 \%)$ had carotid plaques $(C P)$ and the CACscore of these patients was 283.4. In patients without CP CACscore was 53,2 ( $\mathrm{p}<0.05)$.

Based on the presence of CP we performed ROC curve with CACscore. The AUC was 0.778 . The best cut off point was 28 with a sensibility of $71 \%$ and a specifity of $82 \%$. Kappa's coefficient was 0.54 . Twenty patients $(46.5 \%)$ had CACscore $>28$. Compared to patients with CACscore $<28$ statistical significance was found on CIMTmax $(0.93$ vs $0.80 \mathrm{~mm} ; \mathrm{p}<0.01)$, presence of CP $(79 \%$ vs $25 \%, \mathrm{p}<0.01)$ and number of CP $(2.55$ vs $0.43, \mathrm{p}<0.01)$. There was no statistical significance on CIMTm (0.71 vs $0.64 \mathrm{~mm}$ p: 0.11 ).

\begin{tabular}{lc}
\hline Female & $37(82 \%)$ \\
Age & $59(33-83)$ \\
Disease duration & $19(3-57)$ \\
Limited cutaneous SSc & $32(74,4 \%)$ \\
Diffuse cutaneous SSc & $10(23,3 \%)$ \\
Sine Scleroderma SSc & $1(2,3 \%)$ \\
Arterial Hypertension & $26(60,5 \%)$ \\
Diabetes mellitus & $5(11,6 \%)$ \\
Statin treatment & $22(51,2 \%)$ \\
Smokers & $5(11.6 \%)$ \\
Digital ulcers & $24(55,8 \%)$ \\
Pulmonary Hypertension & $6(14 \%)$ \\
Interstitial lung disease & $20(46.5 \%)$ \\
Altered conduction in Electrocardiogram & $15(34,9 \%)$ \\
Left Ventricular diastolic dysfunction $^{\star *}$ & $32(74,4 \%)$ \\
Left ventricular systolic dysfunction & $3(7 \%)$
\end{tabular}

${ }^{*}$ Mean pulmonary artery pressure $>25 \mathrm{~mm} \mathrm{Hg}$ at right heart catheterization. ${ }^{* \star}$ Measured by echocardiography. 
If the presence of $\mathrm{CP}$ or $\mathrm{CAC}$ score $>28$ were considered as subclinical atheromatosis disease (SAD), a total of 26 patients $(60.5 \%)$ were diagnosed. We performed multivariate regression analysis, CIMTm, CIMTmax, low High Density Lipid (HDL), high erythrocyte sedimentation rate (ESR) and age were independent factors for the presence of SAD.

Conclusions: SSc patients often have SAD which is misdiagnosed by CV risk charts. Plaque detection by carotid ultrasonography and CT CACscore are useful to detect SAD. The optimal cut-off point of CACscore in this study is 28. SAD detection would be indicated in elderly, patients with low HDL or high ESR. The measure of CIMT could be useful in some cases.

Disclosure of Interest: None declared

DOI: 10.1136/annrheumdis-2017-eular.1515

\section{SAT0341 PILOT STUDY: HOME MANAGEMENT OF ILOPROST IN THE MICROPUMP/24 HOURS, IN PATIENTS SUFFERING FROM SCLERODERMA, FOLLOWED FOR 12 MONTHS}

G. Pistone, R. Daita, C. Arcuri, N. Catalano, S. Liuzza, C. Rinollo, G. D’Aguanno, A. Allotta, M. Lo Monaco, R. Mallaci Bocchio, G. Natoli, S. Corrao. AZ.OSP.ARNAS Civico, PALERMO, Italy

Background: evaluate the effectiveness on Raynaud's phenomenon and on digital ulcers, safety and side effects, during intravenous infusion of iloprost therapy in patients with diffuse and limited scleroderma

Objectives: the effectiveness of the therapy was assessed at 0 and 12 months, on the basis of daily number of episodes of Raynaud's phenomenon, evaluation of digital ulcers changes of the vascular nail bed to capillaroscopio, evaluation of pulmonary hypertension.

Security according to the appearance of adverse events

Methods: They were enrolled consecutively, for a period of 12 months, 12 patients, 9 women and 2 men, 9 suffering from diffuse scleroderma (positive Scl70) and 3 suffering from limited scleroderma (positive anticentromere), middle age 52,91 years, mean age of disease 7,8 years, 11 non-smoking. They were subjected to infusion of iloprost $1 / 2$ vial diluted in $25 \mathrm{cc}$ of saline solution to 0,6 $\mathrm{ml} / \mathrm{h}$, in micropump Infonde for 24 consecutive hours, for 4 days a month for 12 months, 10 patients with peripheral lines and 2 with central venous access.

Results: of 12 patients with the Raynaud's phenomenon, there was a reduction in the daily number in 4 patients, unchanged in 8 patients. Digital ulcers disappeared in 6 patients, they were unchanged in 5 patients, improved although present in 1 patient. In 7 patients capillaroscopic framework remained unchanged, in 5 patients improved capillary density. 10 patients had no pulmonary hypertension, 2 patients had pulmonary hypertension, no change in the two groups at T0 and T 12. None of the patients experienced side effects during the infusion.

Conclusions: the cyclical infusion therapy iloprost prevented the onset of ulcers in patients at TO not presented or the appearance of new ulcers; it has contributed to the reduction in the daily number of Raynaud's phenomenon and also allowed to reach the same effective dose of a vial of iloprost to dilute in $50 \mathrm{cc}$ of saline solution, allowing the patient to perform at home therapy with greater compliance. In terms of safety none of the patients experienced side effects or adverse effects Disclosure of Interest: None declared

DOI: 10.1136/annrheumdis-2017-eular.5990

\section{SAT0342 TRANSTHORACIC ECHOCARDIOGRAPHY TO QUANTIFY PULMONARY VASCULAR RESISTANCE IN PATIENTS WITH SYSTEMIC SCLEROSIS}

S. Billet ${ }^{1}$, T. Chollet ${ }^{1}$, P. Fournier ${ }^{1}$, L. Tetu ${ }^{2}$, G. Prévot ${ }^{2}$, M. Galinier ${ }^{1}$, D. Carrié ${ }^{1}$, O. Lairez ${ }^{1}$, G. Pugnet ${ }^{3}{ }^{1}{ }^{1}$ Service de Cardiologie, CHU Rangueil; ${ }^{2}$ Service de Pneumologie, CHU Larrey; ${ }^{3}$ Service de Médecine Interne, CHU Toulouse Purpan, Toulouse, France

Background: One of the major causes of systemic sclerosis (SSc)-related death is pulmonary arterial hypertension, which develops in $12-15 \%$ of patients with SSc and accounts for $30-40 \%$ of deaths. Consequently, monitoring of pulmonary arterial pressure (PAP) and resistances is essential in patients with SSc. Abbas formula performed by transthoracic echocardiography (TTE) was reported as a good tool to quantify pulmonary vascular resistances (PVR).

Objectives: Explore the accuracy of TTE and Abbas formula to quantify PVR in patients with SSc.

Methods: All consecutive patients with SSc, diagnosed according to the 2013 ACR/EULAR criteria, or the LeRoy and Medsger criteria for diffuse or limited subsets classification, had within $24 \mathrm{H}$ a Doppler echocardiographic examination and right-heart catheterization were performed. The ratio of peak tricuspid regurgitant velocity (TRV, $m s$ ) to the right ventricular outflow tract time-velocity integral $\left(\mathrm{TVI}_{\mathrm{RVOT}}, \mathrm{cm}\right.$ ) obtained by Doppler echocardiography (TRV/TVI RVOT $_{\text {) }}$ ) was then correlated with invasive PVR measurements using regression analysis. An equation was modeled to calculate PVR in Wood units (WU) using echocardiography, and the results were compared with invasive PVR measurements [1].

Results: Thirty-three consecutive patients were included, 13 (39.4\%) were male and the mean age was $64.6 \pm 12.1$ years. Most were classified as limited cutaneous SSc (IcSSc; $\mathrm{n}=29,87.9 \%$ ). All patients tested positive for antinuclear antibodies, $18(21.2 \%)$ for anti-scleroderma-70, 7 (54.5\%) for anticentromere antibodies and
$2(6.1 \%)$ for anti-RNA polymerase III antibodies. Mean and systolic PAP were $31 \pm 9$ and $53 \pm 16 \mathrm{mmHg}$ respectively. There was a good correlation between right ventricle to right atrium gradient pressure assessed by TTE and $\mathrm{RHC}(\mathrm{R}=0.620$, $\mathrm{P}<0.001)$. RVP assessed by Abbas formula $(2.6 \pm 1.0$ wood units) were well correlated with RVP assessed by RHC (4.8 $2.3 ; \mathrm{R}=0.446, \mathrm{P}=0.013)$.

Figure. Correlations between right ventricular to right atrium gradient and pulmonary arterial resistances assessed by transthoracic echocardiography and right heart catheterization
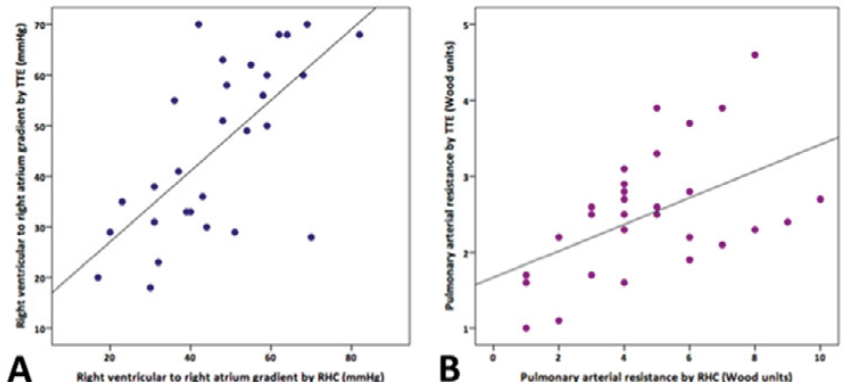

Conclusions: Doppler echocardiography using Abbas formula may provide a reliable, noninvasive method to determine PVR in SSc patients.

\section{References:}

[1] Abbas AE, Fortuin FD, Schiller NB, Appleton CP, Moreno CA, Lester SJ. A simple method for noninvasive estimation of pulmonary vascular resistance. $J$ Am Coll Cardiol. 2003 Mar 19;41(6):1021-7.

Disclosure of Interest: None declared

DOI: 10.1136/annrheumdis-2017-eular.2081

\section{SAT0343 SCREENING OF PULMONARY ARTERIAL HYPERTENSION IN PATIENTS WITH SYSTEMIC SCLEROSIS USING DETECT ALGORITHM - VALIDATION IN THE COHORT OF JAPANESE SINGLE CENTER}

H. Yasuoka ${ }^{1}$, Y. Shirai ${ }^{2}$, Y. Tamura ${ }^{3}$, T. Takeuchi ${ }^{1}$, M. Kuwana ${ }^{2} .{ }^{1}$ Division of Rheumatology, Department of Internal Medicine, Keio University School of Medicine; ${ }^{2}$ Departmenrt of Allergy and Rheumatology, Nippon Medical School Graduate School of Medicine; ${ }^{3}$ International University of Health and Welfare Mita Hospital, Tokyo, Japan

Background: Pulmonary arterial hypertension (PAH) complicated with systemic sclerosis (SSc) has the worst prognosis in $\mathrm{PAH}$ associated with other connective tissue diseases (1) and is one of leading cause of death in patients with SSc (2). To improve prognosis in SSc patients, earlier detection and diagnosis of $\mathrm{PAH}$ by annual screening is recommended even in asymptomatic patients (3). To effectively detect PAH in patients with SSc at earlier phase, DETECT algorithm is reported as a good tool to identify candidates who need right heart catheterization $(\mathrm{RHC})$, with high sensitivity (4). However, its usefulness has not been validated in Japanese cohorts.

Objectives: To validate the effectiveness of DETECT algorithm in the Japanese single center cohort.

Methods: Patients with SSc who visited Keio University Hospital between 2005 and 2016 were included in the study. Patients over 18 years old, disease duration more than 3 years, and DLCO predicted less than $60 \%$ were selected and clinical information was retrospectively collected from records. The sensitivity, specificity, and negative and positive predictive values of the algorithm based on the result of $\mathrm{RHC}$ evaluation were calculated in a cohort of $\mathrm{PAH}$ patients and non-PAH patients, in whom $\mathrm{RHC}$ data were available. Validation with patients with data minimally-required for algorithm were also examined.

Results: Three hundred four cases were visited our hospital during from 2005 to 2016. Patients who fulfilled criteria and had data minimally-required for algorithm were 126 cases. Of 126 patients, 50 were examined $\mathrm{RHC}$ evaluation and patients diagnosed as pulmonary hypertension were $26(21 \%)$ and $21(15 \%)$ were $\mathrm{PAH}$. When a cohort of $\mathrm{PAH}$ patients and non-PAH patients with $\mathrm{RHC}$ data was applied to DETECT algorithm, referral rate to $\mathrm{RHC}$ evaluation was $78 \%$, missed diagnosis of $\mathrm{PAH}$ was $0 \%$. Sensitivity/specificity for detecting PAH patients were $100 \% / 42 \%$, and positive/negative predictive values were $60 \% / 100 \%$, respectively. Evaluation of 126 patients with data minimally-required for algorithm was also examined. Referral rate to $\mathrm{RHC}$ evaluation was $43 \%$, missed diagnosis of $\mathrm{PAH}$ was $0 \%$, and sensitivity/specificity and positive/negative predictive values for detecting $\mathrm{PAH}$ patients were $100 \% / 72 \%, 43 \% / 100 \%$, respectively. However, there was a patient who was initially excluded at Step 1, but developed PAH one year later.

Conclusions: The DETECT algorithm was reassured as a good tool to effectively screening tool for $\mathrm{PAH}$ in SSc patients. However, we have to keep in mind that unnecessity of $\mathrm{RHC}$ referral judged by this algorithm does not guarantee the patient to be free from future development of PAH.

References:

[1] Condliffe R et al. Am J Respir Crit Care Med 179:151-157, 2009.

[2] Steen VD et al. Ann Rheum Dis 66:940-944, 2007 